\title{
Closed Form Aliasing Probability For Q-ary Symmetric Errors
}

\author{
GEETANI EDIRISOORIYA* \\ Motorola Computer Group, MD: DW278, 2900 S. Diablo Way, Tempe, AZ 85282
}

\begin{abstract}
In Built-In Self-Test (BIST) techniques, test data reduction can be achieved using Linear Feedback Shift Registers (LFSRs). A faulty circuit may escape detection due to loss of information inherent to data compaction schemes. This is referred to as aliasing. The probability of aliasing in Multiple-Input Shift-Registers (MISRs) has been studied under various bit error models. By modeling the signature analyzer as a Markov process we show that the closed form expression derived for aliasing probability previously, for MISRs with primitive polynomials under q-ary symmetric error model holds for all MISRs irrespective of their feedback polynomials and for group cellular automata signature analyzers as well. If the erroneous behaviour of a circuit can be modelled with q-ary symmetric errors, then the test circuit complexity and propagation delay associated with the signature analyzer can be minimized by using a set of $\mathrm{m}$ single bit LFSRs without increasing the probability of aliasing.
\end{abstract}

Keywords: Built-In Self-Test, Aliasing probability, LFSR, MISR, Cellular automata, Q-ary symmetric channel

\section{INTRODUCTION}

A digital circuit can be tested by applying an appropriate set of test vectors to its inputs and comparing the actual response of the circuit with the correct or desired response. Testing can be performed using two main approaches: External Test and Built-In Self-Test (BIST). External testing is usually performed by removing the circuit under test from its operational environment and using external testing devices. BIST schemes provide on-chip circuitry necessary to generate test vectors and to analyze output responses so that testing can be performed on site without using external testing devices. Multiple-Input-Shift-Registers (MISRs) are often used as parallel signature analyzers (compactors) in various BIST schemes, to reduce the volume of output data [1]. The final content of the compactor (MISR) is called the signature of the test response, and depends on the bit pattern applied at the input. Errors are detected by comparing the signature with the fault-free signature of the logic circuit. It is possible for a fault to escape detection even though the output bit sequence differs from the fault-free response. This will occur whenever a faulty

*Phone: (602) 438-3090 Fax: (602) 438-3836 Email: geetani@med.mot.com 
output bit sequence generates the fault-free signature of the logic circuit. This effect is known as aliasing or masking. Aliasing may occur with any compaction technique since there are usually many fewer bits in the compacted attribute. The probability of aliasing $\mathrm{P}_{\mathrm{al}}$, depends on the error pattern probabilities.

Aliasing in single input linear feedback shift registers (LFSRs) and MISRs have been studied by several researchers [2-16] under various bit error models to compute $\mathrm{P}_{\mathrm{al}}$. In this work we use the q-ary error model which considers correlated errors among Circuit Under Test (CUT) outputs $[10,15,16]$. The q-ary error model assumes that all $\left(2^{\mathrm{m}}-1\right)$ error patterns possible at the m-output CUT are equally likely. Pradhan et al. [16] argued that q-ary error model is more realistic than the independent bit error model since most real world VLSI circuits have considerable sharing of logic between the outputs. In [16] a closed form expression for computing exact aliasing probability is derived for m-bit MISRs with primitive feedback polynomials and for any test length. Iwasaki and Arakawa [15] showed that the aliasing probabilities over a q-ary symmetric channel do not depend on the polynomials that characterize the MISRs. More general results were obtained in [10]. By modeling the signature analyzer as a Markov process we develop a closed form expression for exact.aliasing probability for all MISRs irrespective of their feedback polynomials and for a class of linear cellular automata (group cellular automata) signature analyzers under q-ary error model. Finally we show that if the output error patterns of a faulty CUT can be approximated by the q-ary error model, then the propagation delay due to feedback path can be minimized by using a set of $m$ single bit LFSRs without increasing the probability of aliasing.

The paper is organized as follows. In section (2) we derive a closed form expression for aliasing probability for MISRs and group cellular automata signature registers using the q-ary symmetric error model. Section (3) presents the register structure with minimum complexity that falls into the class of signature registers considered in this work. Finally in section (4) we conclude with a brief summary.

\section{EXACT ALIASING PROBABILITY}

Figure (1) shows the block diagram of an m-bit MISR connected to a logic circuit. The MISR of Figure (1) is a linear system, thus an error pattern leads to aliasing if and only if the error pattern compacts to the all-0 signature $[2,3]$. In general a MISR with $\mathrm{m}$ cells can be expressed as a polynomial of the following form:

$$
F(X)=f_{0}+f_{1} X+f_{2} X^{2}+\cdots+--f_{m} X^{m}
$$

where $f_{i}=1$ if there is a feedback connection to cell $R_{i}$ and $f_{i}=0$ otherwise. Now any state $R_{j}$ of a MISR can be represented by its binary code $\left(R_{0, j}\right.$, $R_{1, j}, \ldots . . R_{m-1, j}$ ) or by an integer $i$, where $i=0,1,2, .$. , $2^{\mathrm{m}}-1$. Thus, the behaviour of the register can be represented by a stochastic process $\left\{R_{j}, j=0,1,2, . ., 2^{m}\right.$ -1 \}. If $R_{j}=i$, then the process is said to be in state $i$ at time $j$.

The next state of the register depends only upon the present state and does not depend upon previous states. Hence, $\left\{R_{j}, j=0,1,2, . ., 2^{m}-1\right\}$ is a Markov chain. Let $\mathrm{p}_{\mathrm{ij}}$ represent the probability that the process will, when in state $R_{i}$ go to state $R_{j}$ in one step. For the signature register the probability $p_{i j}$ is time invariant. Since probabilities are nonnegative and the process must make a transition into some state,

$$
\sum_{j=0}^{q-1} p_{i j}=1
$$

where $\mathrm{q}=2^{\mathrm{m}}$ and $\mathrm{i}=0,1,2, . ., 2^{\mathrm{m}}-1$.

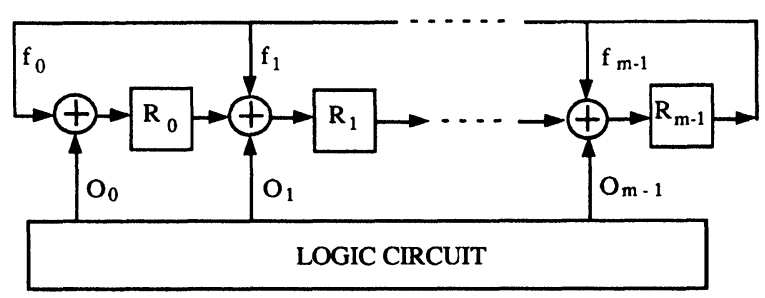

$f_{i}=1$ if there is a connection and 0 otherwise.

FIGURE 1 MISR Compaction. 
Let $\mathrm{P}$ denote the matrix of one-step transition probabilities $\mathrm{p}_{\mathrm{ij}}$, so that

$$
\mathrm{P}=\left(\begin{array}{cccc}
\mathrm{p}_{00} & \mathrm{p}_{01} & \ldots & \mathrm{p}_{0(\mathrm{q}-1)} \\
\mathrm{p}_{10} & \mathrm{p}_{11} & \ldots & \mathrm{p}_{1(\mathrm{q}-1)} \\
\cdot & \cdot & \ldots & \cdot \\
\cdot & \cdot & \ldots & \cdot \\
\mathrm{p}_{(\mathrm{q}-1) 0} & \mathrm{p}_{(\mathrm{q}-1) 1} & \ldots & \mathrm{p}_{(\mathrm{q}-1)(\mathrm{q}-1)}
\end{array}\right)
$$

From Chapman-Kolmogorov equations, $\mathrm{P}^{(\mathrm{k})}=\mathrm{P}^{\mathrm{k}}$. That is, the k-step transition matrix can be obtained by multiplying the matrix $\mathrm{P}$ by itself $\mathrm{k}$ times.

Let $P_{j}(N)$ be the probability of the register being in state $\mathrm{j}$ after $\mathrm{N}$ clock periods starting from the all zero state. Assume that initially no errors are present in the MISR. Then the initial state of the MISR can be represented by the all zero state. Then,

$$
\begin{aligned}
& P_{0}(0)=1 \text { and } \\
& P_{j}(0)=0 \text { where } j=1,2, \ldots ., 2^{m}-1
\end{aligned}
$$

Now the following expression can be used to obtain $P_{j}(N)$, assuming all zero initial state.

$$
\left[\mathrm{P}_{0}(\mathrm{~N}) \mathrm{P}_{1}(\mathrm{~N}) \ldots \ldots . . \mathrm{P}_{\mathrm{q}-1}(\mathrm{~N})\right]=\left[\begin{array}{lllll}
1 & 0 & 0 & \ldots . & 0
\end{array}\right] \mathrm{P}^{\mathrm{N}}
$$

Hence, $\mathrm{P}_{0}(\mathrm{~N})$ the probability of getting all zero state after $\mathrm{N}$ clock periods is given by,

$$
P_{0}(N)=\left[\begin{array}{lllll}
1 & 0 & 0 & \ldots . . .0
\end{array}\right] \mathrm{P}^{\mathrm{N}-1} \mathrm{Q}
$$

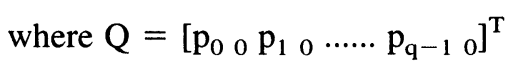

According to the q-ary symmetric error model $[10,15,16]$ all $\left(2^{\mathrm{m}}-1\right)$ error patterns possible at the $\mathrm{m}$-output circuit are equally likely.

THeORem (1): $\quad$ Suppose the MISR is in state $R_{u}$ and it goes to state $R_{v}$ in one clock period in the absence of an error at the output (in the autonomous mode). If the probability of getting an error at the output for any given test input is $p$, then,

$$
\begin{aligned}
p_{u j} & =1-p \text { for } j=v \\
& =\frac{p}{2^{m}-1} \text { otherwise }
\end{aligned}
$$

where $j \in\left\{0,1,2, \ldots . ., 2^{\mathrm{m}}-1\right\}$

Proof: According to the MISR compaction scheme shown in Figure (1), the value of each register is sensitive to the corresponding CUT output. Suppose the register is in state $R_{u}$ and it goes to state $R_{v}$ in one clock period. Let $\left(\mathrm{O}_{0}, \mathrm{O}_{1}, \ldots, \mathrm{O}_{\mathrm{m}-1}\right)$ be the error pattern generated by the CUT. Now state $\mathrm{j}$ is given by,

$$
\begin{gathered}
\mathrm{R}_{\mathrm{v}} \equiv\left(\mathrm{R}_{\mathrm{m}-1, \mathrm{u}} \oplus \mathrm{O}_{0}, \mathrm{R}_{0, \mathrm{u}} \oplus \mathrm{f}_{1} * \mathrm{R}_{\mathrm{m}-1, \mathrm{u}} \oplus \mathrm{O}_{1}, \cdots\right. \\
\left.\cdots, \mathrm{R}_{\mathrm{m}-2, \mathrm{u}} \oplus \mathrm{f}_{\mathrm{m}-1} * \mathrm{R}_{\mathrm{m}-1, \mathrm{u}} \oplus \mathrm{O}_{\mathrm{m}-1}\right)
\end{gathered}
$$

Since each element of $R_{v}$ depends upon a CUT input, the MISR goes to a unique state depending on the error pattern and the current state. Since there are $2^{\mathrm{m}}-1$ non-zero error patterns with equal probability, and the probability of no-error is $1-p$,

$$
\begin{aligned}
\mathrm{p}_{\mathrm{uj}} & =1-\mathrm{p} \text { for } \mathrm{j}=\mathrm{v} \\
& =\frac{\mathrm{p}}{2^{\mathrm{m}}-1} \text { otherwise where } \mathrm{j} \in\left\{0,1,2, \ldots . ., 2^{\mathrm{m}}-1\right\}
\end{aligned}
$$

This proves theorem (1).

If the MISR is initially fault-free, it will remain in the fault-free state in the absence of an erroneous CUT output. Therefore,

$$
\begin{aligned}
& \mathrm{p}_{00}=1-\mathrm{p} \text { and } \\
& \mathrm{p}_{0 \mathrm{j}}=\frac{\mathrm{p}}{2^{\mathrm{m}}-1} \text { otherwise }
\end{aligned}
$$

where $\mathrm{j} \in\left\{1,2, \ldots . ., 2^{\mathrm{m}}-1\right\}$

For example consider the MISR characterized by $\mathrm{F}(\mathrm{X})=1+\mathrm{X}^{2}$ (Figure (2)), and the state transition diagram of the Markov process (Figure (3)). For the q-ary symmetric error model the one-step transition matrix corresponding to $1+\mathrm{X}^{2}$ is given by, 


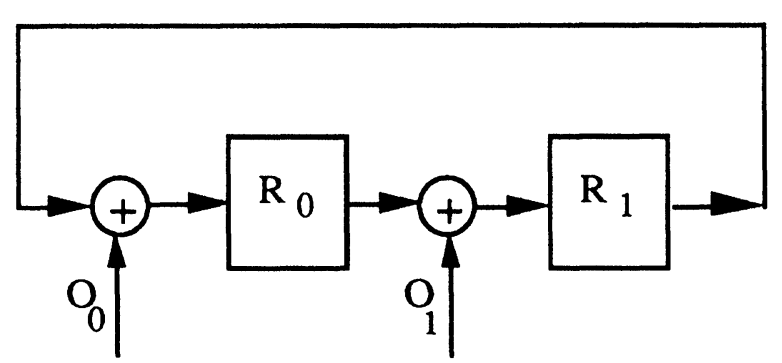

FIGURE 2 MISR corresponding to $1+\mathrm{X}^{2}$.

$$
\mathrm{P}=\left[\begin{array}{cccc}
1-\mathrm{p} & \mathrm{p} / 3 & \mathrm{p} / 3 & \mathrm{p} 3 \\
\mathrm{p} / 3 & \mathrm{p} / 3 & 1-\mathrm{p} & \mathrm{p} / 3 \\
\mathrm{p} / 3 & 1-\mathrm{p} & \mathrm{p} / 3 & \mathrm{p} / 3 \\
\mathrm{p} / 3 & \mathrm{p} / 3 & \mathrm{p} / 3 & 1-\mathrm{p}
\end{array}\right]
$$

According to the q-ary symmetric error model the term $1-p$ is present exactly once in each and every row of the state transition matrix (theorem (1)). It is interesting to note that for the MISR shown in Figure (2) the term $1-p$ is present exactly once in each and every column as well (equation (9)). Consequently, the one-step transition matrix of the MISR characterized by the polynomial $1+X^{2}$ is doubly stochastic. In general, this is true for any MISR defined by equation (1) (see Theorem (2)).

THEOREM (2): The one-step state transition matrices of all MISRs defined by equation (1) are doubly stochastic.

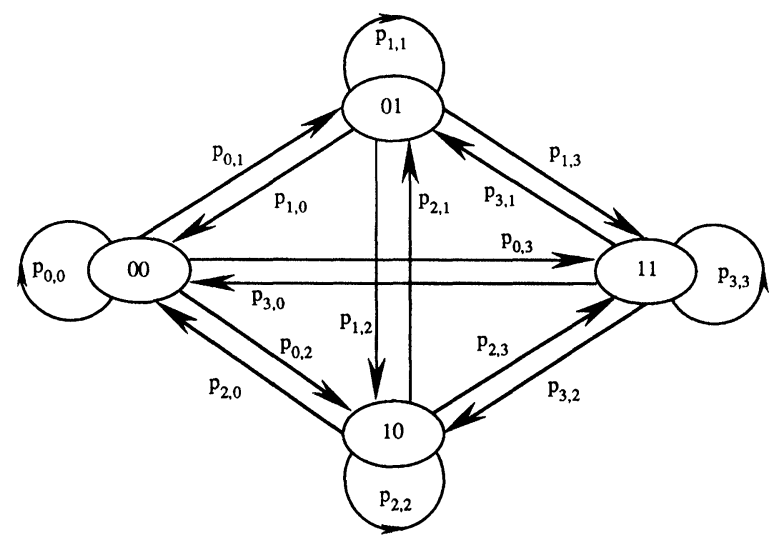

FIGURE 3 State transition diagram of the Markov process for 1 $+\mathrm{X}^{2}$.
Proof: According to the q-ary symmetric error model, for any $\mathrm{i}, \mathrm{j} \in\left\{0,1,2, \ldots . ., 2^{\mathrm{m}}-1\right\}$, $\mathrm{P}_{\mathrm{ij}}=\left\{1-\mathrm{p}, \frac{\mathrm{p}}{\left(2^{\mathrm{m}}-1\right)}\right\}$

In order to prove that the one-step transition matrix is doubly stochastic, we show that each and every column of the state transition matrix contains exactly one $1-p$ term (rest of the elements are $\frac{\mathrm{p}}{\left(2^{\mathrm{m}}-1\right)}$, as $\left.1-\mathrm{p}+\left(2^{\mathrm{m}}-1\right) * \frac{\mathrm{p}}{\left(2^{\mathrm{m}}-1\right)}=1\right)$.

Consider the MISR shown in Figure (1). We can represent state $R_{i}$ of the MISR by its binary code as shown below.

$$
\mathrm{R}_{\mathrm{i}} \equiv\left(\mathrm{R}_{0, \mathrm{i}}, \mathrm{R}_{1, \mathrm{i}}, \ldots . ., \mathrm{R}_{\mathrm{m}-1, \mathrm{i}}\right)
$$

To prove that there is only one $1-p$ term in each column of the state transition matrix, we have to show that if the outputs of the CUT are fault free then the register goes to a unique state depending on the current state. In other words if the current state of the register is $R_{i}$, and it goes to state $R_{j}$ in one step, then the register cannot go to state $R_{j}$ from a state other than $R_{i}$ when the outputs of the CUT are fault free.

Suppose the register is in state $R_{i}$ and it goes to state $R_{j}$ in one step in the absence of faults at the outputs of the CUT. Then we can obtain the state $R_{j}$ from the following expression.

$$
\begin{gathered}
\mathrm{R}_{\mathrm{j}} \equiv\left(\mathrm{R}_{\mathrm{m}-1, \mathrm{i}}, \mathrm{R}_{0, \mathrm{i}} \oplus \mathrm{f}_{1} * \mathrm{R}_{\mathrm{m}-1, \mathrm{i}}, \ldots \ldots,\right. \\
\left.\mathrm{R}_{\mathrm{m}-2, \mathrm{i}} \oplus \mathrm{f}_{\mathrm{m}-1} * \mathrm{R}_{\mathrm{m}-1, \mathrm{i}}\right)
\end{gathered}
$$

Now assume that the state $R_{j}$ can also be reached from state $R_{k}$ in one clock period in the absence of faults at the outputs of the CUT. Then we can obtain the state $R_{j}$ from the following expression.

$$
\begin{gathered}
\mathrm{R}_{\mathrm{j}} \equiv\left(\mathrm{R}_{\mathrm{m}-1, \mathrm{k}}, \mathrm{R}_{0, \mathrm{k}} \oplus \mathrm{f}_{1} * \mathrm{R}_{\mathrm{m}-1, \mathrm{k}}, \cdots \ldots,\right. \\
\left.\mathrm{R}_{\mathrm{m}-2, \mathrm{k}} \oplus \mathrm{f}_{\mathrm{m}-1} * \mathrm{R}_{\mathrm{m}-1, \mathrm{k}}\right)
\end{gathered}
$$

From equations (11) and (12), 


$$
\begin{aligned}
& \mathrm{R}_{\mathrm{m}-1, \mathrm{i}}=\mathrm{R}_{\mathrm{m}-1, \mathrm{k}} \text { and } \\
& \mathrm{R}_{\mathrm{r}, \mathrm{i}} \oplus \mathrm{f}_{\mathrm{r}+1} * \mathrm{R}_{\mathrm{m}-1, \mathrm{i}}=\mathrm{R}_{\mathrm{r}, \mathrm{k}} \oplus \mathrm{f}_{\mathrm{r}+1} * \mathrm{R}_{\mathrm{m}-1, \mathrm{k}}
\end{aligned}
$$

where $\mathrm{r}=0,1, \ldots \ldots, \mathrm{m}-2$.

Therefore

$$
\mathrm{R}_{\mathrm{n}, \mathrm{i}}=\mathrm{R}_{\mathrm{n}, \mathrm{k}}
$$

where $\mathrm{n}=0,1, \ldots \ldots, \mathrm{m}-1$.

and hence

$$
\mathrm{R}_{\mathrm{i}} \equiv \mathrm{R}_{\mathrm{k}}
$$

This proves that in the absence of errors at the outputs of the CUT, the register goes to a unique state depending on the current state.

The probability of aliasing, $\mathrm{P}_{\mathrm{al}}$ is defined as the probability of the MISR initially in the all zero state leaving the all zero state and returning to it [3]. Therefore the probability of aliasing $\mathrm{P}_{\mathrm{al}}$ is given by,

$$
\mathrm{P}_{\mathrm{al}}=\mathrm{P}_{0}(\mathrm{~N})-(1-\mathrm{p})^{\mathrm{N}}
$$

THEOREM (3): Under the q-ary symmetric error model, for any m-input MISR, $P_{0}(N)$ is given by,

$$
P_{0}(N)=\frac{1}{2^{m}}\left[1+\frac{\left[2^{m}(1-p)-1\right]^{N}}{\left(2^{m}-1\right)^{N-1}}\right]
$$

Proof: According to theorem (2) the state transition matrix of any MISR defined by equation (1) is doubly stochastic. Thus, term $1-p$ is present exactly once in each and every row and column of the state transition matrix.

From equation (6),

$$
\begin{array}{r}
P_{0}(\mathrm{~N})=\left[\begin{array}{llll}
1 & 0 & 0 & \ldots . .0
\end{array}\right] \mathrm{P}^{\mathrm{N}-1} \mathrm{Q} \\
\text { Let } \mathrm{P}^{\mathrm{N}-1}=\left(\frac{\mathrm{p}}{2^{\mathrm{m}}-1}\right)^{\mathrm{N}-1} \mathrm{~T}^{\mathrm{N}-1}
\end{array}
$$

Since $\mathrm{P}$ has exactly one $1-\mathrm{p}$ term in each and every row and column (rest of the elements are equal to $\left.\frac{\mathrm{p}}{2^{\mathrm{m}}-1}\right)$, T has exactly one $\frac{(1-\mathrm{p})\left(2^{\mathrm{m}}-1\right)}{\mathrm{p}}$ term in each and every row and column. Also, the remaining elements of $\mathrm{T}$ are equal to 1 . Also since $\mathrm{p}_{00}=1-\mathrm{p}$, $\mathrm{t}_{00}=\frac{(1-\mathrm{p})\left(2^{\mathrm{m}}-1\right)}{\mathrm{p}}$.

From equations (6) and (15),

$$
P_{0}(\mathrm{~N})=\left(\frac{\mathrm{p}}{2^{\mathrm{m}}-1}\right)^{\mathrm{N}-1}\left[\begin{array}{llllll}
1 & 0 & 0 & \ldots \ldots & \ldots
\end{array}\right] \mathrm{T}^{\mathrm{N}-1} \mathrm{Q}
$$

Next we compute $\left[\begin{array}{lllll}1 & 0 & 0 & \ldots \ldots & 0\end{array}\right] \mathrm{T}^{\mathrm{N}-1}$.

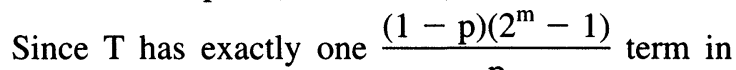
each and every row and column, and the rest of the elements are equal to 1 ,

$$
\begin{gathered}
{\left[\begin{array}{ccccc}
1 & 0 & 0 & \cdots & \ldots . .0
\end{array}\right] \mathrm{T}^{\mathrm{k}-1}} \\
=\left[g_{\mathrm{k}-1}+\mathrm{h}_{\mathrm{k}-1} \mathrm{~h}_{\mathrm{k}-1} \mathrm{~h}_{\mathrm{k}-1} \cdots \cdots-\mathrm{h}_{\mathrm{k}-1}\right]
\end{gathered}
$$

Therefore,

$$
\begin{gathered}
{\left[g_{k}+h_{k} h_{k} h_{k} \cdots-h_{k}\right]} \\
=\left[g_{k-1}+h_{k-1} h_{k-1} h_{k-1} \cdots-h_{k-1}\right]^{*} T
\end{gathered}
$$

Hence,

$$
\begin{gathered}
g_{k}+h_{k}=\left(g_{k-1}+h_{k-1}\right) \frac{(1-p)\left(2^{m}-1\right)}{p} \\
+h_{k-1}\left(2^{m}-1\right)
\end{gathered}
$$

and

$$
\begin{aligned}
h_{k}=\left(g_{k-1}+\right. & \left.h_{k-1}\right)+h_{k-1} \frac{(1-p)\left(2^{m}-1\right)}{p} \\
& +h_{k-1}\left(2^{m}-2\right)
\end{aligned}
$$

From (17) and (18), 


$$
g_{k}=g_{k-1}\left(\frac{2^{m}(1-p)-1}{p}\right)
$$

Using the initial condition $\mathrm{g}_{0}=1$ and the recursive equation (19),

$$
\mathrm{g}_{\mathrm{k}}=\left(\frac{2^{\mathrm{m}}(1-\mathrm{p})-1}{\mathrm{p}}\right)^{\mathrm{k}}
$$

From equations (18) and (20),

$$
h_{k}=\left(\frac{2^{m}(1-p)-1}{p}\right)^{k-1}+h_{k-1} \frac{2^{m}-1}{p}
$$

Using the initial condition $\mathrm{h}_{0}=0$ and the recursive equation (21),

$$
h_{k}=\frac{1}{2^{m}}\left[\left(\frac{2^{m}-1}{p}\right)^{k}-\left(\frac{2^{m}(1-p)-1}{p}\right)^{k}\right]
$$

Since $Q=\left[1-p \frac{p}{2^{m}-1} \cdots-\frac{p}{2^{m}-1}\right]^{T}$,

$$
\begin{aligned}
P_{0}(N) & =\left(\frac{p}{2^{m}-1}\right)^{N-1}\left[g_{N-1}(1-p)+h_{N-1}\right] \\
& =\frac{1}{2^{m}}\left[1+\frac{\left[2^{m}(1-p)-1\right]^{N}}{\left(2^{m}-1\right)^{N-1}}\right]
\end{aligned}
$$

This proves theorem (3).

From equation (14) and theorem (3) for any m-input MISR aliasing probability $\mathrm{P}_{\mathrm{al}}$ is given by,

$$
\begin{aligned}
P_{a l} & =\frac{1}{2^{m}}\left[1+\frac{\left[2^{m}(1-p)-1\right]^{N}}{\left(2^{m}-1\right)^{N-1}}\right]-(1-p)^{N} \\
& =\frac{1}{2^{m}}\left[1-2^{m}(1-p)^{N}+\left(2^{m}-1\right)\left(1-\frac{2^{m} p}{2^{m}-1}\right)^{N}\right]
\end{aligned}
$$

According to definition given in [14], every state for a group cellular automata register has one and only one predecessor. Therefore the one-step state transition matrix of a group cellular automata signature ana- lyzer is doubly stochastic. Also the next state of such a signature register depends upon the current state and the error pattern. Hence, group cellular automata signature registers satisfy theorems (1) and (2). Thus equation (23) which gives the aliasing probability of any MISR, holds for group cellular automata as well.

\section{MINIMUM COMPLEXITY MISR}

Consider the set of $\mathrm{m}$ single bit LFSRs shown in Figure (4).

The state transition matrix of the set of $\mathrm{m}$ single bit LFSRs is given by,

$$
P=\left(\begin{array}{ccc}
1-p \frac{p}{q-1} & \cdots & \frac{p}{q-1} \\
\frac{p}{q-1} 1-p & \cdot \frac{p}{q-1} \\
\cdot & \cdots & \cdot \\
\frac{p}{q-1} \frac{p}{q-1} & \cdots & 1-p
\end{array}\right)
$$

All the diagonal elements of the above transition matrix are equal to $1-p$, and the rest of the elements are equal to $\mathrm{p} /(\mathrm{q}-1)$. Since the $1-\mathrm{p}$ term is present exactly once in each and every row and column of the state transition matrix, and satisfies equation (8), the equation (23) can again be used to obtain aliasing probability of the above structure.

We next show that the network of Figure (4) has the minimum number of exclusive OR operations. Examining the proof of equation (23) we find two necessary conditions:

1) The MISR has m binary state variables

2) The $m$ data sequences being compacted can always change the current state to any of the $2^{\mathrm{m}}$ possible states in one time step.
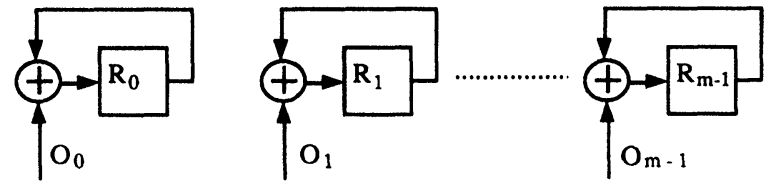

FIGURE 4 Set of $\mathrm{m}$ single bit LFSRs. 
According to condition (1) at least m binary memory elements are required. Also if condition (2) holds, then the next state value for any state variable must depend on at least one test data sequence being compacted. If some state variable were independent of all the test data inputs then at most $2^{\mathrm{m}-1}$ next states would be possible. The minimum dependence would be one test input per state variable and since feedback is required the network of Figure (4) has the minimum number of exclusive-OR gates.

From integrated circuit point of view Figure (4) has minimum propagation delay and a regular structure.

\section{DISCUSSION AND CONCLUSIONS}

In this paper we examined aliasing in MISRs and a class of linear cellular automata (group linear cellular automata) signature analyzers, under an error model based on q-ary symmetric channel. We showed that the expression obtained by Pradhan et al. [16] for aliasing probability is not only applicable to MISRs with primitive feedback polynomials but also applicable to any MISR irrespective of the feedback polynomial and for the class of group automata signature analyzers as well. Finally we showed that if the erroneous behaviour of a circuit can be modelled with q-ary symmetric errors, then the test circuit complexity and propagation delay associated with the signature analyzer can be minimized by using a set of $m$ single bit LFSRs without increasing the probability of aliasing. This also leads to a regular register structure.

\section{References}

[1] C. S. Gloster and Franc Brglez, "Boundary Scan with Built-In Self Test", IEEE Design and Test Magazine, Vol. 6, No. 1, pp. 36-44, February 1989.

[2] J. E. Smith, "Measures of the Effectiveness of Fault Signature Analysis," IEEE Transactions on Computers, Vol. C-29, No. 6, pp. 510-514, June 1980.

[3] T. W. Williams and W. Daehn, "Aliasing Errors in Multiple Input Signature Analysis Registers", Proc. European Test Conf., pp. 338-345, April 1989.

[4] A. Ivanov and V. Agarwal, "On a fast method to Monitor the Behaviour of Signature Analysis Registers", Proc. Int. Test Conf., pp. 645-655, September 1987.
[5] J. P. Robinson, "Aliasing Probabilities for Feedback Signature Compression of Test Data," IEEE Trans. Computers, Vol. 40, pp. 867-873, July 1991.

[6] Geetani Edirisooriya, "Signature Analysis Testing of VLSI Circuits", Ph.D. Thesis, University of Iowa, August 1992.

[7] G. Edirisooriya and J. P. Robinson, "Cyclic Code Weight Spectra and BIST Aliasing", Journal of Electronic Testing: theory and Applications, Vol. 2, No. 2, pp. 153-163, June 1991.

[8] G. Edirisooriya and J. P. Robinson, "Aliasing Properties of Circular MISRs", Journal of Electronic Testing: theory and Applications, Vol. 4, No. 2, pp. 151-158, May 1993.

[9] G. Edirisooriya and J. P. Robinson, "Exact Closed Form Aliasing Probability in a class of Multiple Input Signature Registers", IEE Electronic Letters, Vol. 27, pp. 1590-1592, August 1991.

[10] G. Edirisooriya and J. P. Robinson, "Aliasing Probability in Multiple Input Linear Signature Automata for Q-ary Symmetric Errors", in Proc. Int. Conference on Computer Design, pp. 352-355, October 1991.

[11] G. Edirisooriya and J. P. Robinson, "Time and Space Correlated Errors in Signature Analysis", Proc. IEEE VLSI Test Symposium, pp. 275-281, April 1993.

[12] G. Edirisooriya and J. P. Robinson, "On the Performance of Signature Registers in the Presence of Correlated Errors", Proc. IEE, Part E, Computers and Digital Techniques, Vol. 139, No. 5, pp. 393-400, September 1992.

[13] R. David, "Comments on Signature Analysis for MultipleOutput Circuits", IEEE Transactions on Computers, Vol. 39, No. 2, pp. 287-288, February 1990.

[14] A. K. Das, D. Saha, A. R. Chowdhury, S. Mirsa and P. P. Chaudhuri "Signature Analyzers Based on Additive Cellular Automata", in Proc. Fault Tolerant Comp. Symposium, pp. 265-272, 1990.

[15] K. Iwasaki and F. Arakawa, "An Analysis of the Aliasing Probability of Multiple-Input Signature Registers in the Case of a $2^{\mathrm{m}}$-ary Symmetric Channel", IEEE Transaction on Computer-Aided Design, Vol. 9, No. 4, pp. 427-438, April 1990.

[16] D. K. Pradhan et al., "Aliasing Probability for Multiple Input Signature Analyzer", IEEE Transactions on Computers, Vol. 39, No. 4, pp. 586-591, April 1990.

\section{Author Biography}

Geetani Edirisooriya received the B.Sc. degree with honors in Electronics and Telecommunication Engineering from University of Moratuwa, Sri Lanka in 1986 and Ph.D. from University of Iowa in 1992. From 1986 to 1987 she worked as an assistant lecturer in the Department of Electrical and Computer Engineering at the Open University of Sri Lanka.

She is currently with Fault Tolerant and High End Systems Group, Motorola, Inc., Tempe, AZ. Her research interests include fault tolerant computing, computer aided design and test of VLSI systems and multiprocessor systems. 

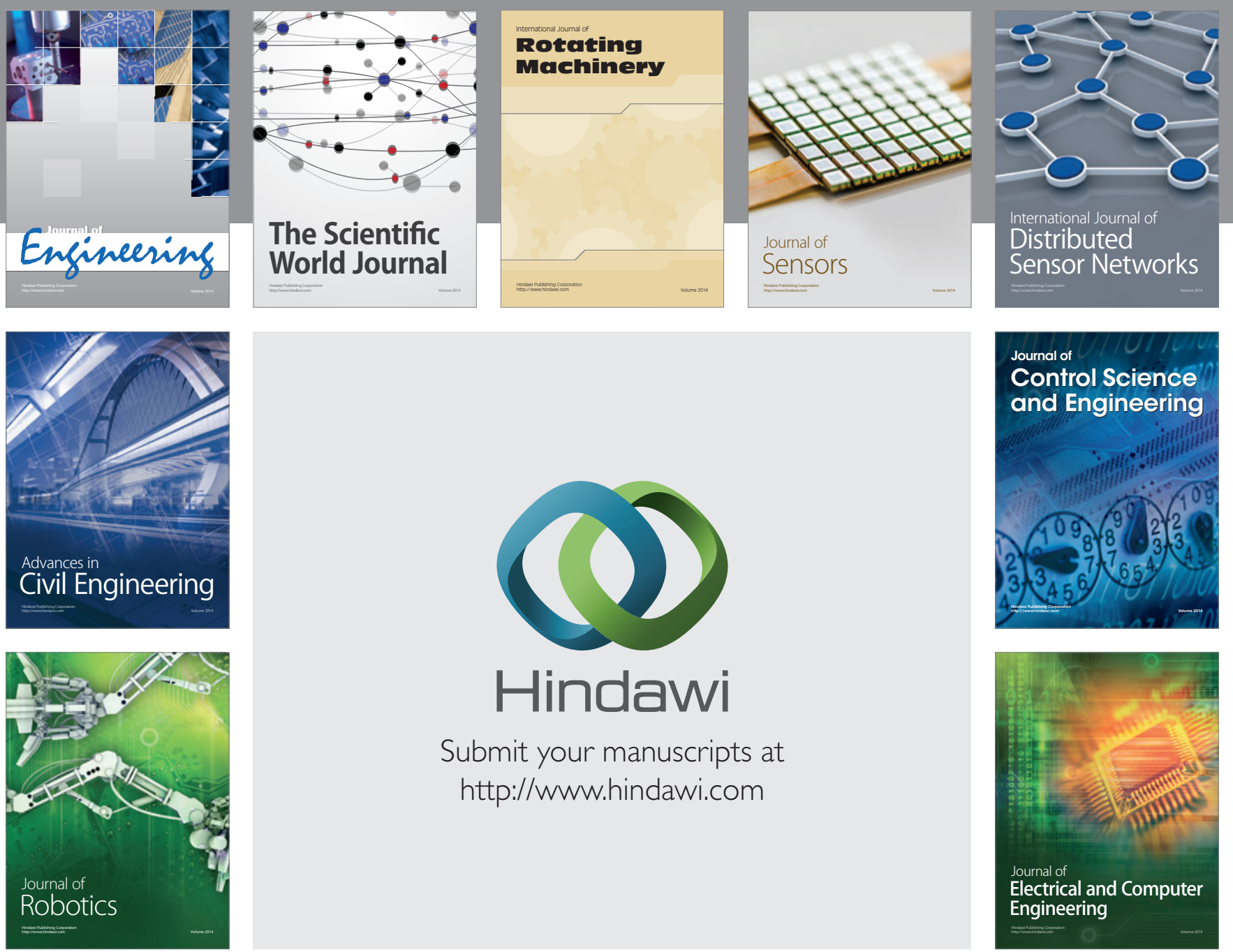

Submit your manuscripts at

http://www.hindawi.com
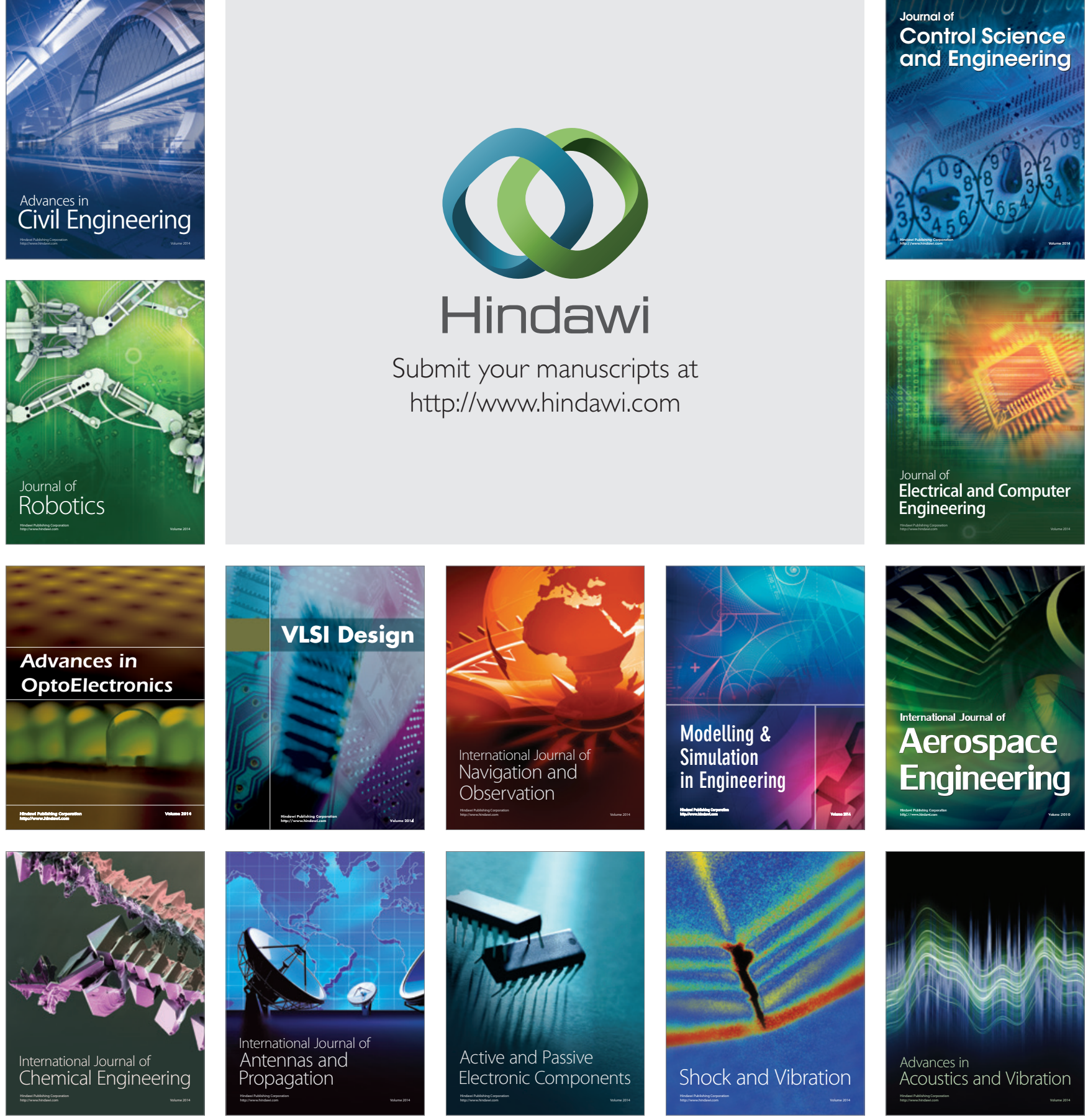